\title{
Global burden of active smoking among people living with HIV on antiretroviral therapy: a systematic review and meta-analysis
}

Boni Maxime Ale ${ }^{1^{*}} \mathbb{D}$, Franck Amahowe ${ }^{1}$, Motto Malea Nganda² ${ }^{2}$ Célestin Danwang ${ }^{3}$, Nelly Njeri Wakaba ${ }^{1}$, Ateeq Almuwallad ${ }^{4,5}$, Franck Biaou Guy Ale ${ }^{1}$, Alamou Sanoussi ${ }^{6}$, Suleiman Hudu Abdullahi ${ }^{7}$ and Jean Joel Bigna ${ }^{8}$

\begin{abstract}
Background: Although the high burden of both active smoking and human immunodeficiency virus (HIV) is clearly known, the relationship between them is still not well characterized. Therefore, we estimated the global prevalence of active smoking in people living with HIV (PLHIV) on antiretroviral therapy (ART) and investigated the association between exposure to active smoking and risk for suboptimal adherence to ART.

Main text: We searched PubMed, Embase, and Web of Science to identify articles published until September 19, 2019. Eligible studies reported the prevalence of active smoking in PLHIV on ART or investigated the association between active smoking and ART adherence; or enough data to compute these estimates. We used a random-effects model to pool data and quantified heterogeneity $\left(I^{2}\right)$. The global prevalence of active smoking was $36.1 \%(95 \% \mathrm{Cl}: 33.7-37.2$; 329 prevalence data; 462104 participants) with substantial heterogeneity. The prevalence increased with level of country income; from 10.1\% (95\% Cl: 6.8-14.1) in low-income to 45.2\% (95\% Cl: 42.7-47.7) in high-income countries; $P<0.0001$. With regards to the Joint United Nations Programme on HIV/AIDS (UNAIDS) regions, the prevalence was higher in West and Central Europe and North America 45.4\% (42.7-48.1) and lowest in the two UNAIDS regions of sub-Saharan Africa: Eastern and Southern Africa 10.7\% (95\% Cl: 7.8-14.0) and West and Central Africa 4.4\% (2.9-6.3); $P<0.0001$. Globally, we estimated that there were 4110669 PLHIV on ART who were active smokers, among which the highest number was from Eastern and Southern Africa (35.9\%) followed by Asia and the Pacific (25.9\%). Active smoking was significantly associated with suboptimal ART adherence: pooled odds ratio 1.57 (95\% Cl: 1.37-1.80; $P^{2}=56.8 \%$; 19 studies; 48450 participants); even after considering adjusted estimates: 1.67 (95\% Cl: 1.39-2.01; $r^{2}=53.0 \% ; 14$ studies).
\end{abstract}

Conclusions: This study suggests a high prevalence of active smoking in PLHIV on ART and an association between active smoking and ART suboptimal adherence. As such, healthcare providers and policy makers should focus on adopting and implementing tobacco harm reduction strategies in HIV care, especially in sub-Saharan Africa known as epicenter of HIV pandemic with highest number of active tobacco smoking among PLHIV on ART.

Keywords: Tobacco, Smoking, HIV, AIDS, Antiretroviral therapy, Global health

*Correspondence: boniale@holo-healthcare.com

${ }^{1}$ Holo Healthcare Limited, Nairobi, Kenya

Full list of author information is available at the end of the article

\section{Background}

Human immunodeficiency virus (HIV) infection remains a global public concern. Globally, there were 37.9 million of people living with HIV (PLHIV) by the end of 2018 [1]. In this population, 24.5 million were accessing 
antiretroviral therapy (ART) [1,2]. To curb the burden of HIV infection, the Joint United Program on HIV/AIDS (UNAIDS) set an ambitious goal that by $2030,95 \%$ of all PLHIV should know their HIV status; $95 \%$ of people diagnosed with HIV infection should receive sustained ART; and $95 \%$ of all people receiving ART should have ribonucleic acid HIV suppression [3]. Since non-adherence to ART is correlated with poor virological response to HIV treatment [4], PLHIV should be voluntary compliant to the prescribed ART to achieve the third 95.

Active tobacco smoking could be one of the factors which would prevent reaching the goal. However, to date, it is not clear whether active smoking could be associated with suboptimal adherence to ART in PLHIV. Globally, tobacco smoking is one of the biggest public health threats the world has ever faced, killing more than 8 million people annually [5]. In addition, tobacco is one of the leading risk factors for cardiovascular diseases, the top killer worldwide [6]. In 2018, 770 000 people died from AIDS-related illnesses $[1,2]$. In addition, HIV infection is now recognized as risk factor of cardiovascular diseases (the top killer worldwide) alongside with traditional cardiovascular risk factors [6].

Although the high burden of both active smoking and HIV is clearly known, the relationship between them and the burden of active smoking in PLHIV on ART are still not well characterized. Accurate global and context-specific epidemiological data are crucial to tackle the burden of active smoking in PLHIV, especially for health-care planning and resource allocations. Hence, we conducted this systematic review with meta-analysis to determine the burden of active smoking in PLHIV undergoing ART. Firstly, we estimated the global prevalence of active smoking in PLHIV on ART and estimated this prevalence by UNAIDS region and country level of income. Secondly, we provided the burden of active smoking in terms of number of smokers in PLHIV on ART at global and UNAIDS regional levels. Finally, we investigated the association between exposure to active smoking and risk for suboptimal adherence to ART in PLHIV.

\section{Methods}

\section{Eligibility criteria}

We used the same method as per already published metaanalyses $[7,8]$. This systematic review and meta-analysis was conducted according to the Joanna Briggs Institute guidelines [9]. This study was reported according to the PRISMA guidelines [10]. The protocol of this review was registered in PROSPERO, CRD42020195796.

We considered observational studies including cross sectional, case-control, and cohort studies. We considered studies reporting the prevalence (or enough data to compute this estimate) of current or active tobacco smoking (willful or deliberate act of inhaling and exhaling smoke from burning substances or agents held by hand) in the global population of PLHIV undergoing ART (at least 90\% of PLHIV with ART) [11]. We also considered studies investigating the association between active tobacco smoking and ART suboptimal adherence in PLHIV.

\section{Search strategy}

We searched PubMed, Excerpta Medica Database (EMBASE), and Web of Science to identify all relevant records published up to September $19^{\text {th }}, 2019$ without any language restriction. The search strategy in EMBASE is available in the Appendix (Supplementary Table 1). This search was adapted to suit with other databases. To supplement the bibliographic database searches and identify potential additional data sources, we scrutinized the reference list of all relevant review papers.

\section{Study selection}

Titles and abstracts of articles retrieved from literature search were independently screened by two investigators, and the full-texts of those potentially eligible were obtained and further assessed for final inclusion. Disagreements were resolved through consensus.

\section{Data extraction and management}

A preconceived and standardized data extraction form was used to collect information on first author's name, study country, year of publication, period of participants' recruitment, study design, setting, sampling method, timing of data collection, response rate, mean or median age of the population, proportion of males, proportion with antiretroviral treatment, number of PLHIV, the number of participants with active smoking. For studies investigating the association between active tobacco smoking and adherence to antiretroviral therapy, we also extracted data for participants with no active smoking, data on the adherence as defined in the original studies, and variables of adjustment in multivariable analysis. Studies were classified by level of income according to the country of recruitment of participants as per World Bank classification [12]. Ten investigators independently extracted data from individual studies, with disagreements being resolved through discussion. Ten investigators independently assessed methodological quality of included studies with tool developed by Joanna Briggs Institute for prevalence data and with Newcastle Ottawa Scale for comparative studies investigating the association between active tobacco smoking and ART adherence 
[9, 13]; with disagreements being resolved through discussion.

\section{Data synthesis and analysis}

Meta-analyses were performed with the meta, metafor, and dmetar packages of the statistical software $R$ (version 3.6.3, $\mathrm{R}$ foundation for Statistical Computing, Vienna, Austria). Prevalence estimates were reported with 95\% confidence interval $(95 \% C I$ ). Prevalence pooling was done with single arcsine transformation using randomeffects meta-analysis model [14]. Sensitivity analyses was performed including only studies with low risk of bias for each of defined items. We estimated an adjusted global prevalence taking in account the variability between UNAIDS region and country level of income. Publication bias was investigated with funnel plot and confirmed with the Egger's test [15]. A $P$-value $<0.10$ on the Egger test was considered indicative of statistically significant publication bias. We performed meta-regression analysis and estimated the variance explained by study and participants' characteristics. In this analysis, the outcome of interest was the prevalence of active smoking in patients undergoing ART, included as a continuous variable. The final multivariable model was chosen based on the lowest corrected Akaike's Information Criterion. For missing data, we have performed a non-parametric test of homoscedasticity and there was not sufficient evidence to reject that data were missing completely at random,
$P=0.450$. Therefore, we have performed multiple-imputation analyses using chained equations for studies who had a missing value. We generated 40 imputed-data sets with a maximum number of 10 iterations, with linear imputation for continuous variables [16]. Variables that were included in the imputation model were proportion of males, mean age, duration of since HIV diagnosis and duration on ART. We have also performed a meta-regression analysis with complete cases.

To measure the association between active tobacco smoking and non-adherence to ART, we did a meta-analysis using the random-effects method to pool weighted (adjusted) odds ratios (OR) of suboptimal ART adherence risk estimate. The symmetry of funnel plot was used to explore publication bias and Harbord test was done to assess the presence of publication bias [17].

Heterogeneity was evaluated by the $\chi^{2}$ test on Cochran's Q statistic [18], which was quantified by $I^{2}$ values. The $I^{2}$ statistic estimates the percentage of total variation across studies due to true difference between-study differences rather than chance. In general, $I^{2}$ values greater than $60-70 \%$ indicate the presence of substantial heterogeneity [19].

\section{Results}

\section{Study selection and characteristics}

Of the 2329 records identified, we included 290 studies with 329 prevalence data for estimating the prevalence

Table 1 Meta-analysis prevalence of active smoking in people living with HIV undergoing antiretroviral therapy

\begin{tabular}{|c|c|c|c|c|c|c|c|c|}
\hline & \multirow[t]{2}{*}{ Prevalence, $\%$} & \multirow{2}{*}{$\begin{array}{l}95 \% \\
\text { confidence } \\
\text { intervals }\end{array}$} & \multirow[t]{2}{*}{ No. studies } & \multirow[t]{2}{*}{ No. participants } & \multicolumn{2}{|c|}{ Heterogeneity } & \multirow[t]{2}{*}{ Egger test } & \multirow[t]{2}{*}{$P$ value, difference } \\
\hline & & & & & $I^{2}, \%$ & Pvalue & & \\
\hline Global & 36.1 & $33.7-38.5$ & 329 & 462104 & 99.5 & $<0.0001$ & 0.0002 & $\ldots$ \\
\hline All participants on ART & 34.5 & $31.8-37.2$ & 268 & 420575 & 99.6 & $<0.0001$ & 0.0001 & $\ldots$ \\
\hline \multicolumn{9}{|l|}{ Country level of income } \\
\hline Low & 10.1 & $6.8-14.1$ & 30 & 11329 & 95.9 & $<0.0001$ & 0.095 & $<0.0001$ \\
\hline Lower-middle & 16.0 & $8.6-25.3$ & 21 & 9858 & 98.7 & $<0.0001$ & 0.496 & \\
\hline Upper-middle & 27.6 & $23.8-31.6$ & 39 & 8252 & 90.4 & $<0.0001$ & 0.668 & \\
\hline High & 45.2 & $42.7-47.7$ & 216 & 339022 & 99.5 & $<0.0001$ & 0.527 & \\
\hline \multicolumn{9}{|l|}{ UNAIDS region } \\
\hline West and Central Africa & 4.4 & $2.9-6.3$ & 12 & 4868 & 87.0 & $<0.0001$ & 0.263 & $<0.0001$ \\
\hline Eastern and Southern Africa & 10.7 & $7.8-14.0$ & 34 & 12676 & 94.8 & $<0.0001$ & 0.080 & \\
\hline $\begin{array}{l}\text { Latin America and the } \\
\text { Caribbean }\end{array}$ & 28.8 & $25.0-32.8$ & 24 & 16385 & 95.9 & $<0.0001$ & 0.162 & \\
\hline Asia and Pacific & 32.6 & $26.6-38.9$ & 27 & 16352 & 97.3 & $<0.0001$ & 0.031 & \\
\hline Middle East and North Africa & 32.9 & $16.1-52.4$ & 3 & 320 & 93.0 & $<0.0001$ & 0.711 & \\
\hline $\begin{array}{l}\text { Eastern Europe and Central } \\
\text { Asia }\end{array}$ & 43.1 & $15.8-72.9$ & 2 & 264 & 95.9 & $<0.0001$ & NA & \\
\hline $\begin{array}{l}\text { West and Central Europe } \\
\text { and North America }\end{array}$ & 45.4 & $42.7-48.1$ & 203 & 293547 & 99.5 & $<0.0001$ & 0.339 & \\
\hline
\end{tabular}


of active smoking in PLHIV on ART and 19 studies for investigating the association between active tobacco smoking and ART adherence (Additional file 1: Figure S1).

Of the 290 studies included in the meta-analysis of prevalence, most of them were cross-sectional, hospitalbased, and published between 2000 and 2019 (Additional file 1: Tables S2 and S3). According to the methodological quality, most of studies used non-probabilistic sampling, had low precision, had unclear description of response rate, were prospectively collected data, and used identical procedure to collect data among participants (Additional file 1: Tables S2 and S3).

Of the 19 studies included in the meta-analysis for the association between active tobacco smoking and adherence to ART, most of them were cross-sectional and hospital-based, had low risk of bias on the selection of participants, included comparable groups and outcome assessment, and were published between 2006 and 2019 (Additional file 1: Tables S4 and S5). All studies used non-probabilistic sampling.

\section{Prevalence of active tobacco smoking in the global population living with HIV}

Of the 329 prevalence data, 216 (65.7\%) were from high, 39 (11.9\%) from upper-middle, 21 (6.4\%) from lower-middle, and 30 (9.1\%) from low-income countries. Twenty-three (7.0\%) were not disagreeable. According to the UNAIDS regional distribution, 203 (61.7\%) prevalence data were from West and Central Europe and North America, 34 (10.3\%) from Eastern and Southern Africa, 27 (8.2\%) form the Asia and Pacific, 24 (7.3\%) from Latin America and the Caribbean, 12 (3.7\%) from West and Central Africa, 3 (0.9\%) from Middle-East and North Africa, and 2 (0.6\%) from Eastern Europe and Central Asia. Twenty-four (7.3\%) prevalence data were multiregional. According to participants' characteristics, the mean or median age varied from 16.7 to 66 years $(n=290)$, the proportion of males varied from 0 to $100 \%$ $(n=301)$, the duration since HIV diagnosis varied from 0.5 to 22 years $(n=119)$, and the duration on ART varied from 0.7 to 18.4 years $(n=98)$.

The global prevalence of active smoking in a total of 462 104 PLHIV undergoing ART was 36.1\% (95\% CI: 33.737.2) with substantial heterogeneity (Table 1). There was asymmetry on the funnel plot corroborated by the Egger test (Additional file 1: Figure S2; Table 1). The prevalence from sensitivity analyses including studies with all patients undergoing ART, and only studies with low risk of bias were close to crude prevalence (Additional file 1: Tables S6). Although in the range of the global crude

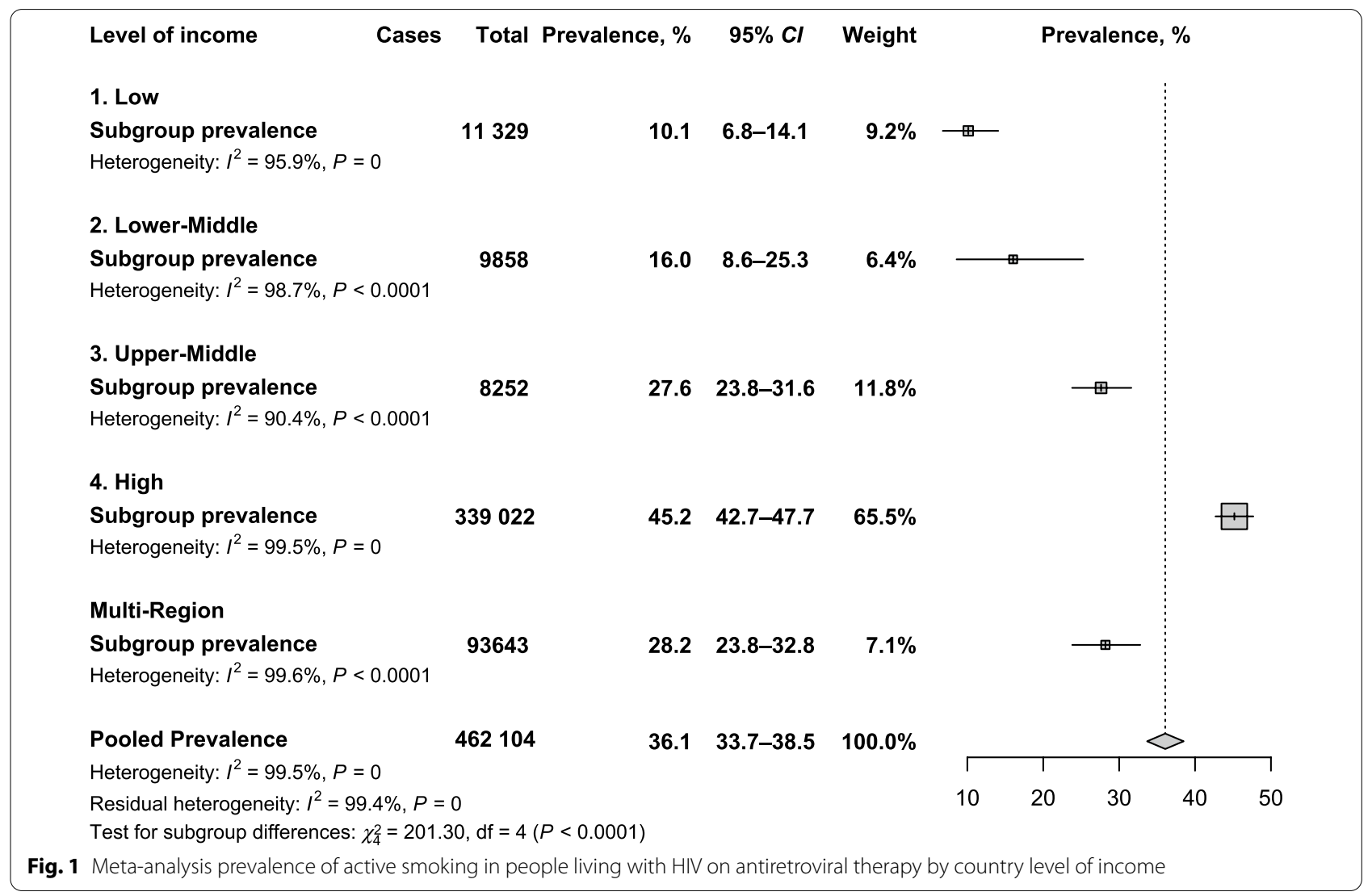




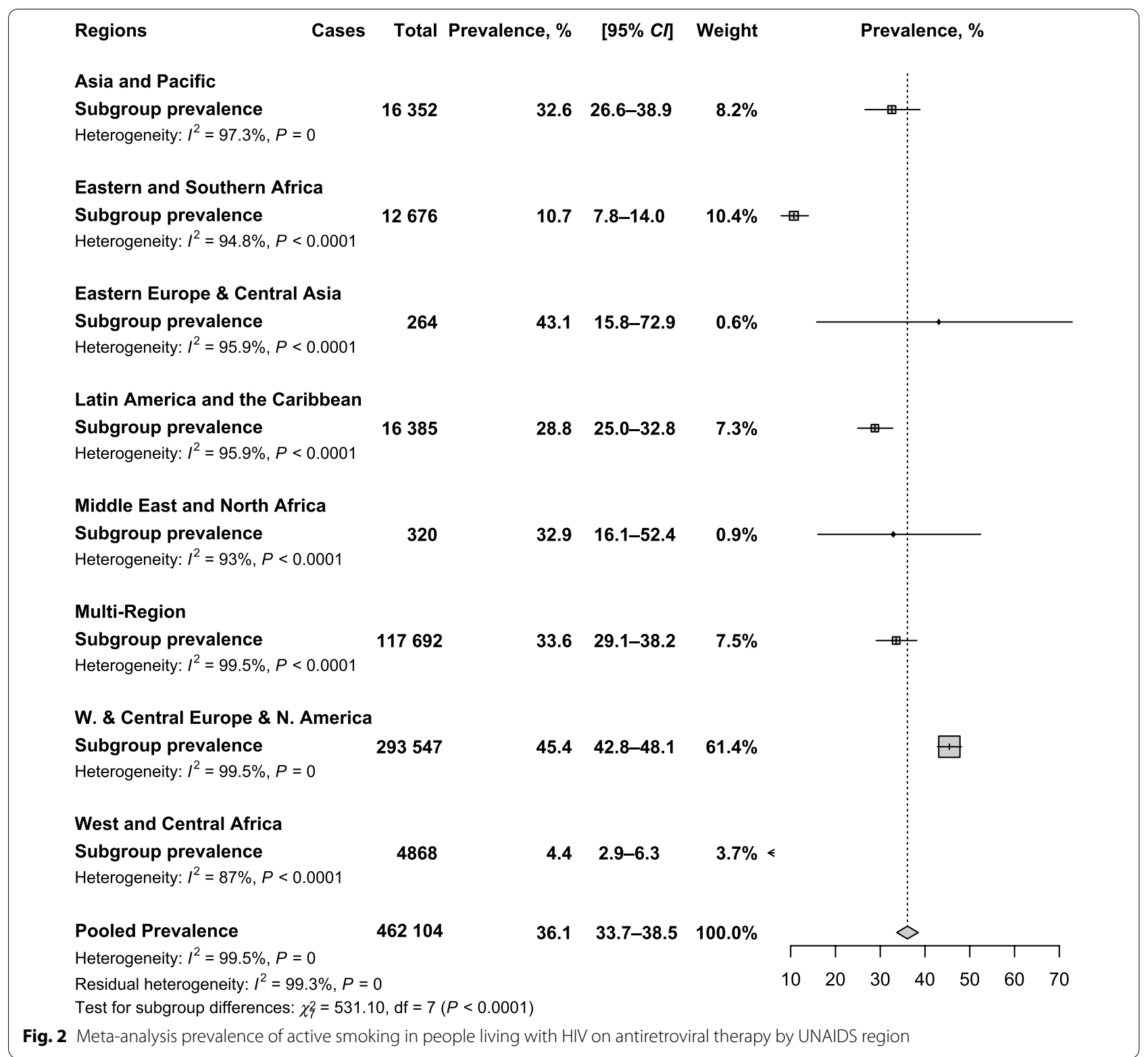

prevalence, the adjusted prevalence taking in account the variance between country level of income and UNAIDS regions was slightly lower: $27.3 \%$ (16.9-39.2).

\section{Sources of heterogeneity of the global prevalence}

In subgroup analysis, the prevalence increased with level of country income; from $10.1 \%(6.8-14.1)$ in low-income countries to $45.2 \%$ (42.7-47.7) in high-income countries, $P<0.0001$ (Fig. 1). According to the UNAIDS region, the lowest prevalence was in sub-Saharan Africa regions: West and Central Africa 4.4\% (2.9-6.9) and Eastern and Southern Africa 10.7\% (7.8-14.0) and the highest prevalence was in West and Central Europe and North America 45.4\% (42.7-48.1), $P<0.0001$ (Fig. 2; Additional file 1:
Figures S3 to S9). Males were more likely to smoke than females: pooled $O R=2.41$ (95\% CI: $1.27-4.60 ; I^{2}=96.3 \%$; 20 studies) (Fig. 3).

In the univariable meta-regression analysis, the explained variance in the global prevalence varied from $0.1 \%$ for response rate to $45.3 \%$ for UNAIDS region (Table 2). The variation in the global prevalence significantly increased with country level of income, proportion of males, duration since HIV infection diagnosis, duration on ART, increasing age, and was associated with UNAIDS region (Table 2). There was no difference with the analysis considering complete cases (Additional file 1: Tables S7).

In the final multivariable meta-regression model, the global prevalence increased with country level of 


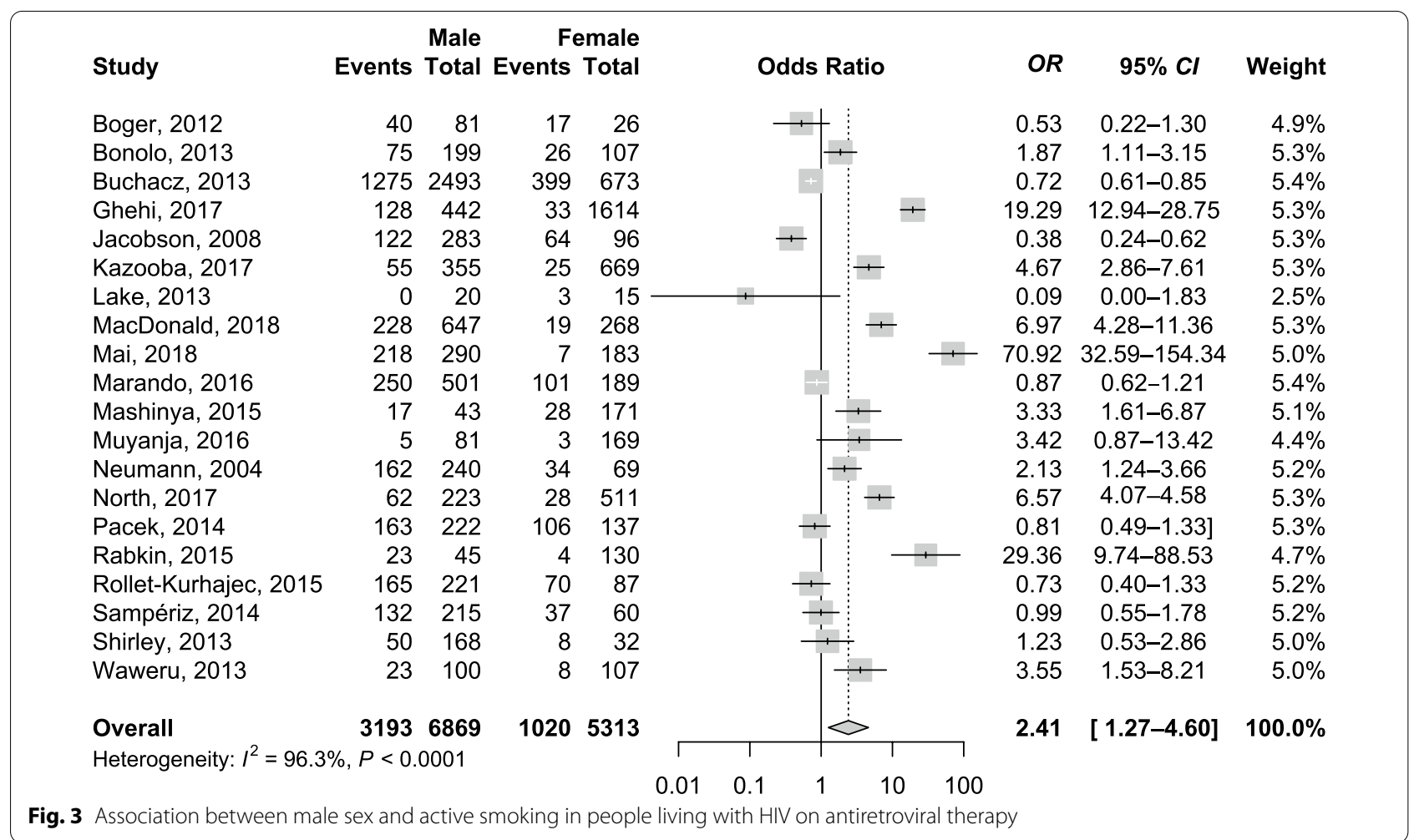

income. The prevalence was lower in Eastern and Southern Africa, in West and Central Africa, in Latin America and the Caribbean compared to West and Central Europe and North America. There was no difference for other regions. These two variables accounted for $47.3 \%$ of the variation in the global prevalence (Table 2). The same variables were included in the final model considering completes cases (Additional file 1: Tables S7).

\section{Burden of active tobacco smoking in people undergoing antiretroviral treatment}

Considering regional estimates, 35.9\% of PLHIV undergoing ART were from Eastern and Southern Africa, followed by Asia and Pacific (25.4\%) and West and Central Europe and North America (18.8\%). Globally, 4110669 PLHIV undergoing ART were active smokers (Table 3).

\section{Active tobacco smoking and adherence to antiretroviral therapy}

We included 19 studies with 48450 PLHIV undergoing ART in the meta-analysis for investigating the association between active tobacco smoking and suboptimal adherence to ART. There was clinical heterogeneity in the definition of adherence to ART across included studies (Additional file 1: Tables S5). The risk for suboptimal adherence to ART was significantly higher in patients with active tobacco smoking than in non-active smoking: pooled $O R=1.57$ (95\% CI: $1.37-1.80 ; I^{2}$ : $56.8 \%$; 19 studies) (Fig. 4a). There was no asymmetry on the funnel plot (Additional file 1: Fig. S10), confirmed by the Harbord test $(P=0.293)$. The meta-analysis of adjusted $O R$ yielded a pooled estimate of 1.67 (95\% CI: $1.39-2.01 ; I^{2}=53.0 \% ; 14$ studies) (Fig. 4b). There was no asymmetry on the funnel plot (Additional file 1: Fig. S11), confirmed by the Harbord test $(P=0.410)$.

\section{Discussion}

In this meta-analysis of 329 prevalence data with 462104 PLHIV on ART, we found that more than third of them actively smoke tobacco. The prevalence of active tobacco smoking in PLHIV in ART was higher in Western \& Central Europe and North America UNAIDS region. However, the burden in terms of number of PLHIV on ART that actively smoke was higher in Eastern and Southern Africa. The prevalence increased with country level of income and was higher among males compared to females. We also found that active smoking was associated with suboptimal ART adherence.

The prevalence of active smoking in PLHIV on ART found in this study is close to the one reported among females in a previous meta-analysis of 51 studies $(36.3 \%$, 95\% CI: $28.0-45.4 \%$ ) and slightly lower compared to the one reported among males (50.3\%, 95\% CI: 44.4-56.2\% 


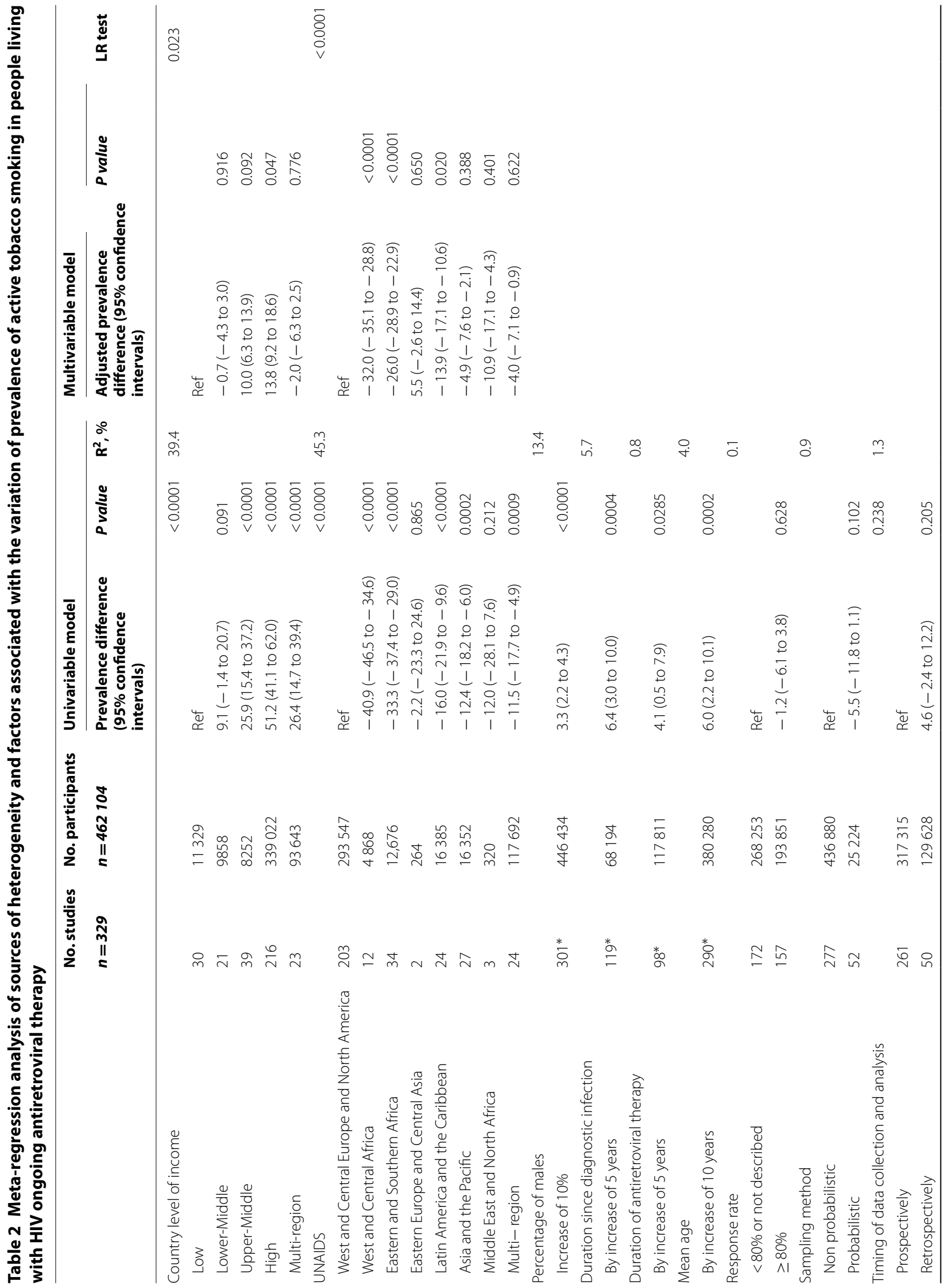




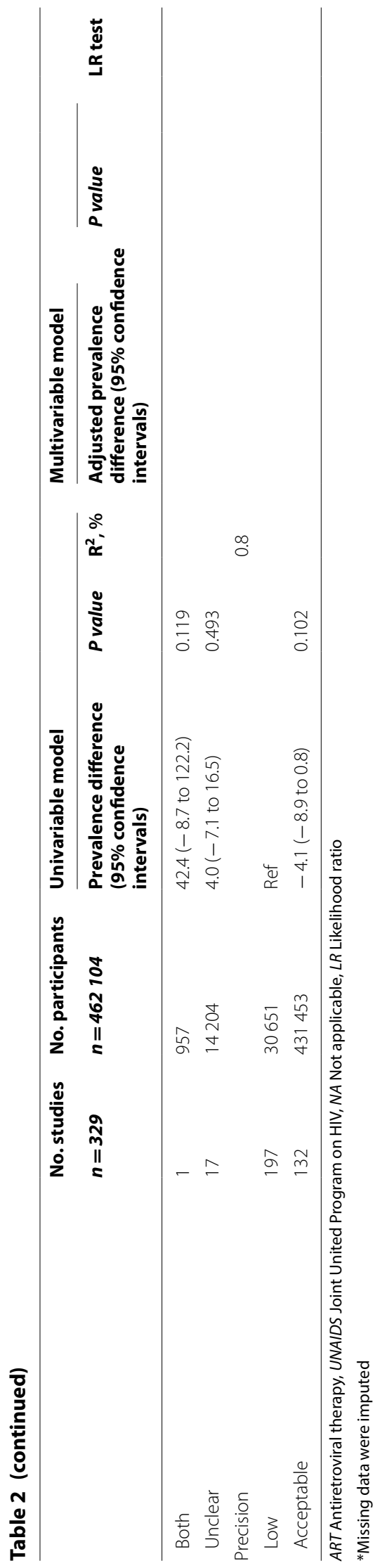


Table 3 Global and regional estimates of active smoking in people living with HIV undergoing antiretroviral therapy

\begin{tabular}{lll}
\hline Regions & $\begin{array}{l}\text { Estimated number of PLHIV undergoing ART } \\
\text { with active smoking }\end{array}$ & $\begin{array}{c}\text { Proportion among total } \\
\text { regional estimable cases }\end{array}$ \\
\hline Global & 4110669 \\
Eastern and Southern Africa & 1476600 \\
Asia and the Pacific & 1043200 \\
West and Central Europe and North America & 771800 \\
Latin America and the Caribbean & 399456 \\
Eastern Europe and Central Asia & $27928.9 \%$ \\
West and Central Africa & 114400 \\
Middle East and North Africa & 25925 & $9.7 \%$ \\
\hline
\end{tabular}

HIV Human Immunodeficiency Virus, PLHIV People living with HIV, ART Antiretroviral treatment

in the same study [20]. Another meta-analysis of 31 studies found a prevalence $46.5 \%$ (95\% CI: 40.9-52.1) among PLHIV regardless of their ART status [21]. Our review included more studies and focused on PLHIV undergoing ART. In addition, our review also represents a more contemporary and global estimate. Like in the general population $[22,23]$, the odds ratio of active smoking was higher among males compared to females in this study. In the global general population, about $40 \%$ of men smoke as compared to nearly $9 \%$ of women [22]. Indeed, results from neuroimaging studies suggest that smoking activates men's reward pathways more than women [24]. This is reflected by higher odds of smoking among men compared to women in this meta-analysis and other metaanalyses of PLHIV regardless of their ART status [20, 21].

The prevalence increased with country level of income. We found a higher prevalence in the Western and Central Europe and North America UNAIDS region (where most of the countries are classified as high-income) and lower in all UNAIDS Africa regions (West and Central Africa and Eastern and Southern Africa, where most of the countries are classified as low and lower-middle income). This might be explained by the fact that tobacco is more affordable for populations with higher socioeconomic status. In addition, people have less chance to smoke in warm-weather context where most of low- and lowermiddle income countries are located [25]. Furthermore, Western \& Central Europe and North America countries might be culturally more prone to smoke. Indeed, in the general population, age-standardized prevalence of tobacco smoking among persons 15 years and older was higher in World Health Organization (WHO) Europe region in 2016 and may partly explain a higher prevalence of active smoking in PLHIV on ART in Western and Central Europe and North America UNAIDS countries [26]. Although, Western and Central Europe and North America UNAIDS region had the highest prevalence of active smoking, the burden in terms of number of active smokers in PLHIV on ART was higher in
Eastern and Southern Africa, which accounted for almost one-third of all PLHIV with active smoking. Hence, prioritization of resources and implementation of contextspecific public health interventions to curb the burden of active smoking in PLHIV should be designed to address sub-Saharan Africa in particular.

We found that the odd of suboptimal ART adherence was significantly higher in active smokers compared to non-active. Evidence indicate that structural and socioeconomic conditions, as well as psychosocial factors (depression, anxiety), that were associated with smoking behavior are also predictive of suboptimal ART adherence. Prior research indicates that smokers, compared with nonsmokers tended to have multiple and interrelated structural and socioeconomic conditions, as well as psychosocial factors, that were associated with suboptimal ART adherence [27]. Improved understanding of the ways in which these factors co-occur and affect health-related behaviors may improve the efforts to decrease the burden of tobacco smoking when integrating adherence interventions into clinical settings.

Our findings have important policy implications for the management of PLHIV on ART because among the 24.5 million PLHIV ongoing ART worldwide [1], more than 4 million are active smokers and may not achieve HIV viral suppression due to suboptimal ART adherence. Both HIV-itself and tobacco smoking are associated with numerous health concerns including but not limited to cancer, cardiovascular diseases, hypertension, lung diseases, diabetes, tuberculosis, immune system diseases, and chronic obstructive pulmonary disease [7, 28-31]. Therefore, curbing the burden of tobacco smoking in PLHIV on ART is paramount and requires efficient interventions against this modifiable behavior and risk factor since ART initiation do not favor quitting smoking [32]. The high active smoking prevalence found in this study calls all HIV healthcare providers to pay more attention to this dreadful public health concern and to integrate quitting tobacco interventions in already built 
a Crude meta-analysis

\begin{tabular}{|c|c|c|c|c|c|c|}
\hline Study & \multicolumn{2}{|c|}{ Log OR S.E. Log OR } & Odds Ratio & OR & $95 \%-\mathrm{Cl}$ & Weigh \\
\hline Aye, 2017 & 0.94 & 0.3517 & \begin{tabular}{c}
-1 \\
\hdashline
\end{tabular} & 2.57 & {$[1.29 ; 5.12]$} & $3.0 \%$ \\
\hline Batista, 2014 & 0.48 & 0.1324 & $\div$ & 1.62 & {$[1.25 ; 2.09]$} & $8.8 \%$ \\
\hline Bonolo [Men], 2013 & -0.03 & 0.3223 & & 0.97 & {$[0.52 ; 1.82]$} & $3.4 \%$ \\
\hline Bonolo [Women], 2013 & 1.42 & 0.4897 & & 4.15 & {$[1.59 ; 10.85]$} & $1.7 \%$ \\
\hline Camargo, 2019 & 0.23 & 0.4459 & & 1.26 & {$[0.53 ; 3.02]$} & $2.0 \%$ \\
\hline Cioe, 2017 & 0.44 & 0.3286 & & 1.55 & {$[0.81 ; 2.95]$} & $3.3 \%$ \\
\hline Cropsey, 2016 & 0.21 & 0.0795 & + & 1.23 & {$[1.06 ; 1.44]$} & $11.2 \%$ \\
\hline Degroote, 2014 & 0.91 & 0.3454 & & 2.49 & {$[1.26 ; 4.90]$} & $3.0 \%$ \\
\hline Jordan, 2014 & 0.81 & 0.3150 & 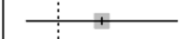 & 2.24 & {$[1.21 ; 4.15]$} & $3.5 \%$ \\
\hline Murri, 2009 & 0.88 & 0.2913 & 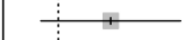 & 2.41 & {$[1.36 ; 4.27]$} & $3.9 \%$ \\
\hline Nguyen, 2016 & 0.05 & 0.1391 & - & 1.05 & {$[0.80 ; 1.38]$} & $8.5 \%$ \\
\hline Nolan, 2017 & 0.66 & 0.4505 & I & 1.93 & {$[0.80 ; 4.67]$} & $2.0 \%$ \\
\hline O'Connor, 2013 & 0.54 & 0.0536 & + & 1.71 & {$[1.54 ; 1.90]$} & $12.1 \%$ \\
\hline Peretti-Watel, 2006 & 0.44 & 0.0822 & $\div$ & 1.55 & {$[1.32 ; 1.82]$} & $11.0 \%$ \\
\hline Sharma, 2013 & 1.23 & 0.5479 & \begin{tabular}{c}
1 \\
\hdashline
\end{tabular} & 3.44 & {$[1.17 ; 10.06]$} & $1.4 \%$ \\
\hline Silva, 2015 & 0.53 & 0.3537 & & 1.70 & {$[0.85 ; 3.40]$} & $2.9 \%$ \\
\hline Soares, 2019 & 0.76 & 0.3231 & \begin{tabular}{l}
$\vdots$ \\
\hdashline
\end{tabular} & 2.13 & {$[1.13 ; 4.01]$} & $3.4 \%$ \\
\hline Winhusen, 2018 & 0.34 & 0.2291 & 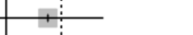 & 1.41 & {$[0.90 ; 2.20]$} & $5.3 \%$ \\
\hline Yuan, 2006 & 0.18 & 0.1163 & 1 & 1.20 & {$[0.95 ; 1.50]$} & $9.5 \%$ \\
\hline \multicolumn{3}{|c|}{ Overall } & $\dot{\theta}$ & \multirow[t]{2}{*}{1.57} & \multirow[t]{3}{*}[1.37;1.80]{} & $100.0 \%$ \\
\hline \multirow{2}{*}{\multicolumn{2}{|c|}{ Heterogeneity: $I^{2}=56.8 \%, p=0.0012$}} & & & & & \\
\hline & & 0. & 2 & 2 & & \\
\hline
\end{tabular}

b Adjusted meta-analysis

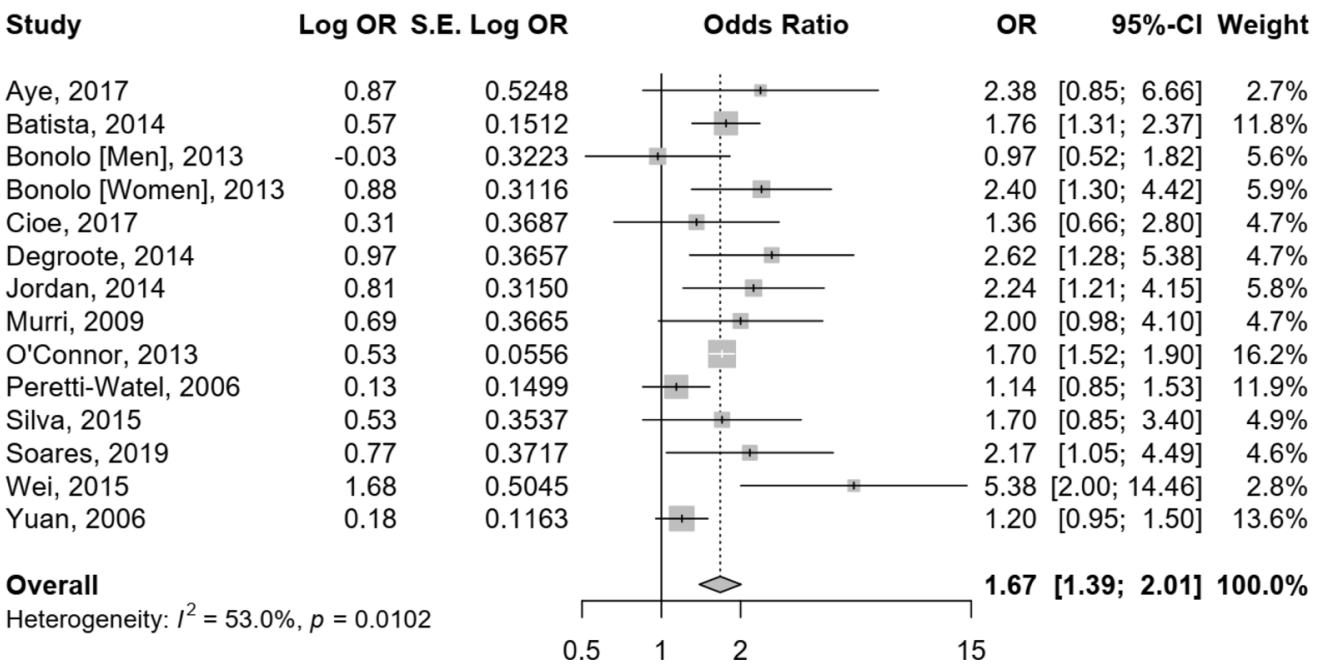

Fig. 4 Association between active smoking and suboptimal adherence to antiretroviral therapy in people living with HIV. a Crude estimate, $\mathbf{b}$ Adjusted estimate

HIV services. All PLHIV with active smoking should be identified and solutions to quit smoking should be implemented including but not limited to mobile phone-based, internet-based, pharmacotherapy-based, nicotine-based, incentives, counseling, and motivational interview interventions [33-44]. Future research should investigate quitting tobacco smoking in the specific population of PLHIV on ART. Countries should fully implement the six MPOWER guidelines issued by the WHO. To date, only Brazil and Turkey have had the highest level of achievement in terms of this guidelines implementation [5].

The findings of this review should be interpreted considering some limitations. First, we included some studies with moderate to high risk of bias. However, when pooling only studies having a low risk of bias, the overall estimate was close to that of the crude analysis. Second, we found a huge statistical heterogeneity between studies for which we undertook subgroup and meta-regression analyses to identify sources of heterogeneity. This is 
most common to meta-analyses of prevalence data [45]. However, some characteristics that may further explain heterogeneity were not consistently reported in the original studies such as HIV clinical stage, immunological status, HIV viral load and detailed ART regimen to further explore other sources of heterogeneity. Third, the various geographic regions and countries were variably represented. This may weaken the generalizability of our findings. To take in account this variability, we performed a hierarchical meta-analysis considering the variance from UNAIDS regions and country level of income. Fourth, there was methodological and clinical heterogeneity on the method to measure ART adherence, and ART regimen information was lacked or was not consistently reported across included studies. Therefore, we were not able to statistically quantify this heterogeneity although this was not substantial when measuring the association between active smoking and ART adherence. Finally, there were some missing data concerning study characteristics for which we have undertaken multiple imputation to address this limitation. Despite these limitations, this systematic review and meta-analysis provided a clear summary of the existing knowledge on the global burden of active smoking among PLHIV on ART at global and regional levels and according to the country level of income. It is also the first measuring the association between active smoking and ART adherence.

\section{Conclusions}

This study suggests a high prevalence of active smoking in PLHIV on ART and an association between active smoking and ART suboptimal adherence. As such, active smoking in PLHIV should be prioritized among HIV health care providers, policy makers and stakeholders from the health sector for improved detection, overall proper management and efficient control. This study supports the need for specific strategies to reduce the burden of active smoking in PLHIV, with primer focus on males and on sub-Saharan Africa, the epicenter of HIV pandemic with the highest number of active smokers among PLHIV on ART.

\section{Supplementary Information}

The online version contains supplementary material available at https://doi. org/10.1186/s40249-021-00799-3.

Additional file 1. Additional figures and tables

\section{Abbreviations}

ART: Antiretroviral therapy; Cl: Confidence interval; HIV: Human immunodeficiency virus; OR: Odds ratio; PLHIV: People living with HIV; UNAIDS: Joint United Program on HIV/AIDS; WHO: World Health Organization.
Acknowledgements

Not applicable.

\section{Authors' contributions}

Conception: BMA and JJB. Design: BMA, AA, FA, FBGA, NW, AS, CD, MMN, SHA, JJB. Search strategy: JJB. Study selection based on title and abstract: BMA and $J$ JB. Study selection based on full text: BMA, AA, FA, FBGA, NW, AS, CD, MMN, SHA, JJB. Data extraction: BMA, AA, FA, FBGA, NW, AS, CD, MMN, SHA, JJB. Data management: JJB. Data synthesis and analysis: BMA and JJB. Data interpretation: BMA and JJB. Manuscript drafting: BMA and JJB. Manuscript revision and review: BMA, AA, FA, FBGA, NW, AS, CD, MMN, SHA, JJB. Approved the final version: All authors. Guarantors of the review: BMA and JJB. All authors read and approved the final manuscript.

Funding

There was no funding for this study.

Availability of data and materials

All data generated or analyzed during this study are included in this published article and its supplementary information files.

Ethics approval and consent to participate

Not applicable.

Consent for publication

Not applicable.

\section{Competing interests}

The authors declare that they have no competing interests.

\begin{abstract}
Author details
${ }^{1}$ Holo Healthcare Limited, Nairobi, Kenya. ${ }^{2}$ Department of Clinical Science and International Public Health, Liverpool School of Tropical Medicine, Liverpool, UK. ${ }^{3}$ Epidemiology and Biostatistics Unit, Institute of Experimental and Clinical Research, Université Catholique de Louvain, Brussels, Belgium.

${ }^{4}$ Applied Medical Sciences College, Jazan University, Jazan, Saudi Arabia.

${ }^{5}$ Center for Trauma Science, Queen Mary University of London, London, UK.

${ }^{6}$ Transition Support Program Department, Advocate Good Samaritan Hospital, Downers Grove, IL, USA. ${ }^{7}$ Leprosy and Tuberculosis Relief Initiative Nigeria, Plateau, Nigeria. ${ }^{8}$ Department of Epidemiology and Public Health, Centre Pasteur of Cameroon, Yaoundé, Cameroon.
\end{abstract}

Received: 24 August 2020 Accepted: 21 January 2021

Published online: 12 February 2021

\section{References}

1. UNAIDS. Global HIV \& AIDS statistics — 2019 fact sheet. 2019. https:// www.unaids.org/en/resources/fact-sheet. Accessed June 292020.

2. UNAIDS. UNAIDS data 2019. 2019. https://www.unaids.org/sites/default/ files/media_asset/2019-UNAIDS-data_en.pdf. Accessed June 292020.

3. UNAIDS. Understanding fast-track: accelerating action to end the AIDS epidemic by 2030. 2015. https://www.unaids.org/sites/default/ files/media_asset/201506_JC2743_Understanding_FastTrack_en.pdf. Accessed June 292020.

4. Nieuwkerk PT, Oort FJ. Self-reported adherence to antiretroviral therapy for HIV-1 infection and virologic treatment response: a meta-analysis. J Acquir Immune Defic Syndr. 2005;38:445-8.

5. WHO. Tobacco: Key facts. 2020. https://www.who.int/news-room/factsheets/detail/tobacco. Accessed June 292020.

6. WHO. Cardiovascular diseases (CVDs): Key facts. 2017. https://www.who. int/en/news-room/fact-sheets/detail/cardiovascular-diseases-(cvds). Accessed June 292020.

7. Bigna JJ, Ndoadoumgue AL, Nansseu JR, Tochie JN, Nyaga UF, et al. Global burden of hypertension among people living with HIV in the era of increased life expectancy: a systematic review and meta-analysis. J Hypertens. 2020;38:1659-68.

8. Leumi S, Bigna JJ, Amougou MA, Ngouo A, Nyaga UF, et al. Global burden of hepatitis B infection in people living with HIV: a systematic review and meta-analysis. Clin Infect Dis. 2019; ciz1170. 
9. JBI: JBI Reviewer's Manual. In. Edited by Aromataris E, Munn Z: Joanna Briggs Institute; 2020.

10. Liberati A, Altman DG, Tetzlaff J, Mulrow C, Gøtzsche PC, loannidis JP, et al. The PRISMA statement for reporting systematic reviews and meta-analyses of studies that evaluate healthcare interventions: explanation and elaboration. BMJ (Clinical research ed). 2009;339:b2700.

11. U.S. National Library of Medicine. MeSH: Smoking. https://www.ncbi.nlm. nih.gov/mesh/68012907. Accessed Jul 212020.

12. World Bank. World Bank Country and Lending Groups. 2020. https:// datahelpdesk.worldbank.org/knowledgebase/articles/906519-world -bank-country-and-lending-groups. Accessed Oct 102020.

13. Wells GA, Shea B, O'Connell D, Peterson J, Welch V, Losos M, Tugwell P. The Newcastle-Ottawa Scale (NOS) for assessing the quality of nonrandomised studies in meta-analyses. 2019. http://www.ohri.ca/programs/ clinical_epidemiology/oxford.asp. Accessed June 62020.

14. Schwarzer G, Chemaitelly H, Abu-Raddad L, Rücker G. Seriously misleading results using inverse of Freeman-Tukey double arcsine transformation in meta-analysis of single proportions. Res Synth Methods. 2019;10:476-83.

15. Egger M, Davey Smith G, Schneider M, Minder C. Bias in meta-analysis detected by a simple, graphical test. BMJ (Clinical research ed). 1997;315:629-34.

16. Graham JW, Olchowski AE, Gilreath TD. How many imputations are really needed? Some practical clarifications of multiple imputation theory. Prev Sci. 2007;8:206-13.

17. Harbord RM, Egger M, Sterne JA. A modified test for small-study effects in meta-analyses of controlled trials with binary endpoints. Stat Med. 2006;25:3443-57.

18. Cochran WG. The combination of estimates from different experiments. Biometrics. 1954;10:101-29.

19. Higgins JP, Thompson SG. Quantifying heterogeneity in a meta-analysis. Stat Med. 2002;21:1539-58.

20. Weinberger AH, Smith PH, Funk AP, Rabin S, Shuter J. Sex differences in tobacco use among persons living with HIV/AIDS: a systematic review and meta-analysis. J Acquir Immune Defic Syndr. 2017;74:439-53.

21. Lindayani L, Yeh C-Y, Ko W-C, Ko N-Y. High smoking prevalence among HIV-positive individuals: a systematic review and meta-analysis. JSubst Use. 2020;25:1-10.

22. WHO. 10 facts on gender and tobacco. 2010. https://www.who.int/gende r/documents/10facts_gender_tobacco_en.pdf. Accessed Jul 122020.

23. Hitchman SC, Fong GT. Gender empowerment and female-to-male smoking prevalence ratios. Bull World Health Organ. 2011;89:195-202.

24. Cosgrove KP, Wang S, Kim SJ, McGovern E, Nabulsi N, Gao H, et al. Sex differences in the brain's dopamine signature of cigarette smoking. J Neurosci. 2014;34:16851-5.

25. Momperousse D, Delnevo CD, Lewis MJ. Exploring the seasonality of cigarette-smoking behaviour. Tob Control. 2007;16:69-70.

26. World Health Organization. Prevalence of tobacco smoking: Agestandardized prevalence of tobacco smoking among persons 15 years and older (\%), by WHO region, 2015. 2015. http://apps.who.int/gho/data/ node.sdg.3-a-viz?lang=en. Accessed Apr 152017.

27. Cioe PA, Gamarel KE, Pantalone DW, Monti PM, Mayer KH, Kahler CW. Cigarette Smoking and Antiretroviral Therapy (ART) Adherence in a Sample of Heavy Drinking HIV-Infected Men Who Have Sex with Men (MSM). AIDS Behav. 2017:21:1956-63.
28. Centers of Disease Control and Prevention. Smoking \& Tobacco Use: Health Effects. 2020. https://www.cdc.gov/tobacco/basic_information/ health_effects/index.htm. Accessed Jul 122020.

29. Bigna JJ, Kenne AM, Asangbeh SL, Sibetcheu AT. Prevalence of chronic obstructive pulmonary disease in the global population with HIV: a systematic review and meta-analysis. Lancet Glob Health. 2018;6:e193-202.

30. Bigna JJ, Sime PS, Koulla-Shiro S. HIV related pulmonary arterial hypertension: epidemiology in Africa, physiopathology, and role of antiretroviral treatment. AIDS Res Ther. 2015;12:36.

31. UNAIDS. HIV and AIDS: Basic facts. 2020. https://www.unaids.org/en/ frequently-asked-questions-about-hiv-and-aids. Accessed Jul 122020

32. Nansseu JR, Tounouga DN, Noubiap JJ, Bigna JJ. Changes in smoking patterns after HIV diagnosis or antiretroviral treatment initiation: a global systematic review and meta-analysis. Infect Dis Poverty. 2020;9:35.

33. Whittaker R, McRobbie H, Bullen C, Rodgers A, Gu Y. Mobile phonebased interventions for smoking cessation. Cochrane Database Syst Rev. 2016:4:Cd006611.

34. Schuit E, Panagiotou OA, Munafò MR, Bennett DA, Bergen AW, David SP. Pharmacotherapy for smoking cessation: effects by subgroup defined by genetically informed biomarkers. Cochrane Database Systematic Rev. 2017:9:Cd011823

35. Notley C, Gentry S, Livingstone-Banks J, Bauld L, Perera R, HartmannBoyce J. Incentives for smoking cessation. Cochrane Database Systematic Rev. 2019;7:CD004307.

36. El Dib R, Suzumura EA, Akl EA, Gomaa H, Agarwal A, Chang Y, et al. Electronic nicotine delivery systems and/or electronic non-nicotine delivery systems for tobacco smoking cessation or reduction: a systematic review and meta-analysis. BMJ Open. 2017;7:e012680

37. Hartmann-Boyce J, Chepkin SC, Ye W, Bullen C, Lancaster T. Nicotine replacement therapy versus control for smoking cessation. Cochrane Database Syst Rev. 2018;5:Cd000146.

38. Cahill K, Stevens S, Perera R, Lancaster T. Pharmacological interventions for smoking cessation: an overview and network meta-analysis. Cochrane Database Syst Rev. 2013:Cd009329.

39. Cahill K, Stead LF, Lancaster T. Nicotine receptor partial agonists for smoking cessation. Cochrane Database Syst Rev. 2007:Cd006103.

40. Stead LF, Koilpillai P, Fanshawe TR, Lancaster T. Combined pharmacotherapy and behavioural interventions for smoking cessation. Cochrane Database Syst Rev. 2016;3:Cd008286.

41. Taylor GMJ, Dalili MN, Semwal M, Civljak M, Sheikh A, Car J. Internetbased interventions for smoking cessation. Cochrane Database Syst Rev. 2017:9:Cd007078

42. Lancaster T, Stead LF. Individual behavioural counselling for smoking cessation. Cochrane Database Syst Rev. 2017:3:Cd001292.

43. Lindson-Hawley N, Thompson TP, Begh R. Motivational interviewing for smoking cessation. Cochrane Database Syst Rev. 2015:Cd006936.

44. Pool ER, Dogar O, Lindsay RP, Weatherburn P, Siddiqi K. Interventions for tobacco use cessation in people living with HIV and AIDS. Cochrane Database Syst Rev. 2016:Cd011120.

45. Bigna JJ, Nansseu JR, Kaze AD, Noubiap JJ. The Authors Respond. Epidemiology. 2018;29:e58-9.

Ready to submit your research? Choose BMC and benefit from:

- fast, convenient online submission

- thorough peer review by experienced researchers in your field

- rapid publication on acceptance

- support for research data, including large and complex data types

- gold Open Access which fosters wider collaboration and increased citations

- maximum visibility for your research: over $100 \mathrm{M}$ website views per year

At BMC, research is always in progress.

Learn more biomedcentral.com/submissions 